\title{
Growth of ZnO Nanostructures with Controllable Morphology Using a Facile Green Antisolvent Method
}

\author{
Ji-Yao Dong, ${ }^{\dagger}$ Yung-Jung Hsu, ${ }^{\dagger}$ David Shan-Hill Wong, ${ }^{*}, \dagger$ and Shih-Yuan Lu*,† \\ Department of Chemical Engineering, National Tsing Hua University, Hsinchu, Taiwan 30013, \\ Republic of China, and Department of Materials Science and Engineering, National Chiao Tung University, \\ Hsinchu, Taiwan 30010, Republic of China
}

Received: March 16, 2010; Revised Manuscript Received: April 7, 2010

\begin{abstract}
An environmentally friendly antisolvent approach, which involves only biocompatible chemicals and mild processing conditions, was developed to prepare various $\mathrm{ZnO}$ nanostructures including twin-cones and nanorods with controllable dimensions. The method was based on the dissolution of $\mathrm{ZnO}$ powders in a deep eutectic solvent (DES), followed by precipitation of $\mathrm{ZnO}$ nanostructures from the DES upon introduction of an antisolvent. It is the first example of the room temperature ionic liquid based antisolvent process for preparation of nanomaterials. Through suitably modulating the processing conditions, such as the ethanol content of the antisolvent and the injection time of the ZnO-containing DES, the morphology of the resulting $\mathrm{ZnO}$ nanostructures can be readily controlled. Anisotropic crystal growth was achieved without severe reaction conditions, such as high temperatures and high vacuums, or use of environmentally harmful chemicals, such as long carbon chain surfactants and capping reagents. The present method can be readily extended to produce versatile nanostructures of other functional materials.
\end{abstract}

\section{Introduction}

Zinc oxide $(\mathrm{ZnO})$ is a wide bandgap $(3.37 \mathrm{eV})$ semiconductor with a large exciton binding energy of $60 \mathrm{meV}$, which makes it a promising material for room temperature light emission. To date, many relevant applications including light emitting diodes, ${ }^{1}$ laser diodes, ${ }^{2}$ optoelectronics, ${ }^{3}$ photocatalysts, ${ }^{4}$ piezoelectrics, ${ }^{5}$ and sensing ${ }^{6}$ have been successfully developed for various nanostructures of $\mathrm{ZnO}$. It is generally agreed that morphology plays an important role in determining the electronic and optical properties of semiconducting materials, including $\mathrm{ZnO}$. A wide variety of synthetic routes have thus been proposed to prepare specific nanostructures of $\mathrm{ZnO}$, including nanorods, ${ }^{7}$ nanowires, ${ }^{8}$ nanobelts, ${ }^{9}$ tetrapods,${ }^{10}$ and many other anisotropic prototypes, to further the development of $\mathrm{ZnO}$. Among the many synthetic approaches, wet chemistry is a low-cost and yet effective way for large-scale productions of $\mathrm{ZnO}$ in liquid phase. In wet-chemistry processes, surfactants and capping reagents are often required to control the crystal growth of the materials to enable formation of anisotropic nanostructures. Meanwhile, through suitably modulating the reactant concentrations, ${ }^{11}$ reaction temperature, type of solvent, ${ }^{12}$ or by applying seed layers, ${ }^{13}$ organic additives, ${ }^{14}$ and quenching treatments ${ }^{15}$ during the reaction, $\mathrm{ZnO}$ nanostructures with controllable dimensions have been obtained. Most of the above operations, however, involve either the use of environmentally harmful chemicals (precursor, surfactant, and solvent) or complicated synthetic procedures, which may hinder the applicability of the products. Therefore, development of a more facile, environmentally friendly synthetic route for $\mathrm{ZnO}$ nanostructures with controllable morphology is crucial to their practical applications. We report here a facile green antisolvent approach, which involves only

\footnotetext{
* Corresponding author. E-mail: dshwong@ @ che.nthu.edu.tw (D.S.-H.W.) sylu@mx.nthu.edu.tw (S.-Y.L.).

National Tsing Hua University.

* National Chiao Tung University.
}

biocompatible chemicals and mild processing conditions, to produce $\mathrm{ZnO}$ nanostructures with controllable morphology. The method uses commercial $\mathrm{ZnO}$ powders as the raw material, a biocompatible room temperature ionic liquid as the solvent, and ethanol/water mixtures as the antisolvent.

Room temperature ionic liquids, possessing many advantageous characteristics, including high polarity, high ionic conductivity, negligible vapor pressure, good thermal stability, and wide liquid windows, are considered a new class of green solvents and may replace the role played by often hazardous organic solvents in many applications. ${ }^{16}$ They have been used extensively in the preparation of nanostructured inorganic materials. ${ }^{17}$ Many of these ionic liquids however are expensive with uncertain biocompatibility. ${ }^{18}$ Deep eutectic solvents (DES), which can be prepared by simply mixing quaternary ammonium salts with proton donors, are a new class of room temperature ionic solvents and are cheap and environmentally friendly. ${ }^{19}$ For example, urea, with a melting point of $133{ }^{\circ} \mathrm{C}$, and choline chloride, with a melting point of $302{ }^{\circ} \mathrm{C}$, can be simply mixed in a 2 to 1 molar ratio to form a DES (denoted as UCC) with a reported melting point of only $12{ }^{\circ} \mathrm{C}$, much lower than those of the two constituents. Both constituents of the UCC are cheap and commonly found in bio-organisms and therefore environmentally benign. Besides, one key characteristic of the UCC is that it is a good solvent for many metal oxides, such as $\mathrm{CuO}$, $\mathrm{NiO}$, and especially $\mathrm{ZnO} .^{20}$ The solvent property of the UCC toward $\mathrm{ZnO}$ will be lost when the solution is mixed with water or ethanol, an antisolvent showing no solvation ability toward $\mathrm{ZnO}$. This would result in the nucleation and growth of $\mathrm{ZnO}$ to lead to the formation of $\mathrm{ZnO}$ nanostructures. In this work, the antisolvent concept was applied to fabricate anisotropic nanostructures of $\mathrm{ZnO}$, including twin-cones and nanorods, with controllable dimensions. This is the first example of using DES as a solvent in an antisolvent process for preparation of functional nanomaterials and a brand new addition to the ever expanding application scope of room temperature ionic liquids. 
The approach was based on the dissolution of $\mathrm{ZnO}$ powders in the UCC DES, followed by the precipitation of $\mathrm{ZnO}$ nanostructures from the $\mathrm{ZnO}$-containing DES upon introduction of the antisolvent. Through suitably modulating the processing conditions, such as the ethanol content of the antisolvent and the injection time of the $\mathrm{ZnO}$-containing DES, the morphology of the resulting $\mathrm{ZnO}$ nanostructures can be readily controlled. The as-obtained products were characterized and analyzed with SEM, XRD, HRTEM, and PL. A plausible mechanism for the growth of the various $\mathrm{ZnO}$ nanostructures is also proposed and discussed.

\section{Experimental Methods}

Preparation of $\mathrm{ZnO}$-Containing DES. All chemicals were used without further purification. An amount of $150 \mathrm{~g}$ of UCC DES was first prepared by mixing $69 \mathrm{~g}$ of urea (Riedel-de Haën, $99.5 \%$ ) and $81 \mathrm{~g}$ of choline chloride (Sigma-Aldrich, 98\%), in a molar ratio of 2 to 1 and in a glovebox with an argon atmosphere. An amount of $0.36 \mathrm{~g}$ of $\mathrm{ZnO}$ powders (SigmaAldrich, 99\%) was then added to the UCC to afford a solution containing roughly $2400 \mathrm{ppm}$ of $\mathrm{ZnO}$. These UCC solutions were kept in an oven at $70{ }^{\circ} \mathrm{C}$ for several days until all $\mathrm{ZnO}$ powders dissolved and clear solutions were obtained.

Growth of $\mathrm{ZnO}$ in Antisolvent Process. Ethanol (SigmaAldrich, 99.8\%) and deionized water were mixed in different volume ratios and used as the antisolvent for $\mathrm{ZnO}$. For the growth of $\mathrm{ZnO}, 5.0 \mathrm{~g}$ of $\mathrm{ZnO}$-containing DES was injected into $200 \mathrm{~mL}$ of antisolvent in $5 \mathrm{~s}$ in a water bath maintained at $70{ }^{\circ} \mathrm{C}$, followed by vigorous stirring for $5 \mathrm{~min}$. The resultant white suspending solid was collected by centrifugation at 6500 rpm for $30 \mathrm{~min}$ and washed with deionized water and ethanol to remove remaining DES and impurities. The product was then dispersed in ethanol for later use. In this work, antisolvents of three different ethanol contents $(0,50$, and 90 vol \%) were employed for the growth of $\mathrm{ZnO}$. Besides, experiments with two different injection times of the $\mathrm{ZnO}$-containing DES (5 s and $5 \mathrm{~h}$ ) were conducted and compared to investigate the effects of the injection time on the morphology of the resulting $\mathrm{ZnO}$.

Characterizations. The morphology and dimensions of the products were examined with a field emission scanning electron microscope (FESEM, Hitachi S-4700) operated at an accelerating voltage of $15 \mathrm{kV}$. The crystallographic structures of the samples were investigated with an X-ray diffractometer (XRD, Rigaku, Ultima IV) and high-resolution transmission electron microscope (HRTEM, JEOL, JEM-3000F) operated at $300 \mathrm{kV}$. The room temperature photoluminescence (PL) spectra were recorded with a fluorescence spectrophotometer (Hitachi, F-4500) using a $150 \mathrm{~W}$ Xe lamp as the excitation source. The excitation wavelength was set at $320 \mathrm{~nm}$.

\section{Results and Discussion}

The structures and dimensions of the as-prepared products were first investigated with SEM and XRD. Figure 1 shows the SEM images and XRD patterns of the $\mathrm{ZnO}$ samples obtained with antisolvents of different ethanol contents. Note that the injection time of the $\mathrm{ZnO}$-containing DES was $5 \mathrm{~s}$, the fast injection case. SEM observations revealed that a large quantity of rice-grain like particles was produced when using pure water as the antisolvent. As shown in Figure 1a, these elliptically shaped particles had a minor-axis length of 300 to $650 \mathrm{~nm}$ and a major-axis length ranging from hundreds of nm to several $\mu \mathrm{m}$. When the antisolvent of $50 \%$ ethanol was applied, a much more delicate twin-cone nanostructure was obtained, as shown in Figure 1b. The twin-cone feature, structurally composed of

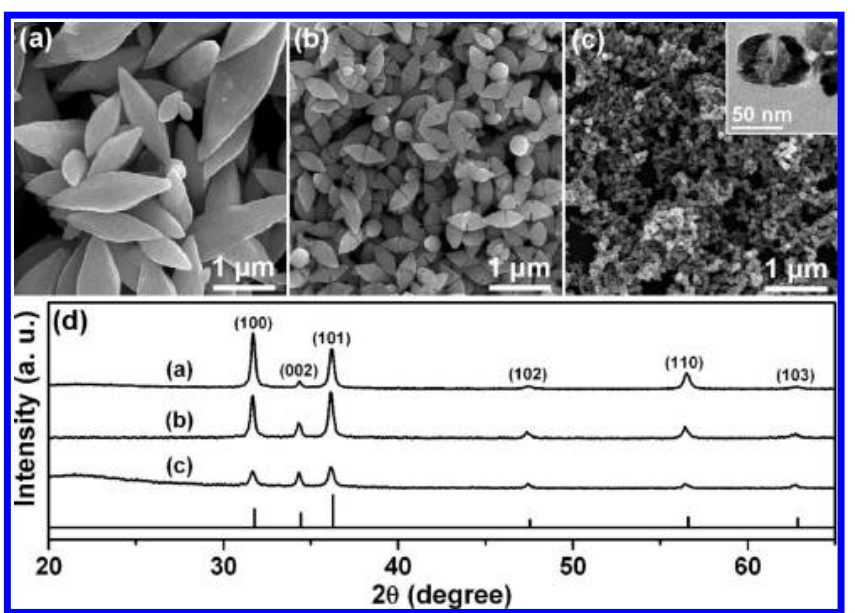

Figure 1. SEM images of $\mathrm{ZnO}$ nanostructures prepared with the antisolvent of (a) pure water, (b) $50 \%$ ethanol and $50 \%$ water, and (c) $90 \%$ ethanol and $10 \%$ water. The inset of (c) is a TEM image of a single twin-cone particle. The corresponding XRD patterns are shown in (d). Injection time of $\mathrm{ZnO}$-containing $\mathrm{DES}=5 \mathrm{~s}$.

two cones with round bottoms connected to each other, can be clearly identified with the well-resolved slits observed at the middle of the particles. These twin cones were with a minoraxis length of 200 to $300 \mathrm{~nm}$ and a major-axis length of 600 to $700 \mathrm{~nm}$, a much better uniformity in shape and dimension than that of the rice-grain like particles obtained with pure water as the antisolvent. Further increase in the ethanol content of the antisolvent to $90 \%$ led to the growth of granular particles with a particle size of about $60 \mathrm{~nm}$. In fact, many of the granular particles preserved the twin-cone-like morphology, as can be more clearly seen from the magnified TEM image in the inset of Figure 1c. The corresponding XRD patterns shown in Figure $1 \mathrm{~d}$ reveal the crystallographic structure of the wurtzite phase for all three $\mathrm{ZnO}$ samples. It should be noted that there were no diffraction peaks attributable to zinc hydroxide or other impurities, indicating the success of the present antisolvent process for production of pure $\mathrm{ZnO}$.

Further structural characterizations were carried out using TEM and HRTEM. Typical TEM images with the corresponding selected-area electron diffraction (SAED) patterns for these three $\mathrm{ZnO}$ samples were shown in Figure $2 \mathrm{a}-\mathrm{c}$. In Figure 2a, if examined closely, a slit can also be identified at the middle of the elliptically shaped particle, indicating that the twin-cone structure is intrinsic to the products obtained with the fast injection practice. The inset SAED patterns suggest that the elliptically shaped and twin-cone particles both substantially grew as single crystals. The dotted-ring SAED pattern of Figure 2c implies that the granular particles may also be single crystalline. Signals from several randomly oriented granular particles covered by the sampling electron beam gave rise to the observed dotted-ring pattern. In addition, the stretched dots of the SAED patterns obtained for the elliptically shaped and twin-cone samples imply the existence of structural defects such as dislocations and planar deformations. ${ }^{21}$ Figure $2 \mathrm{~d}-\mathrm{f}$ further shows the detailed crystallographic structures of the three twincone based samples. The lattice images taken at the marked regions clearly reveal the (0002) lattice planes of the wurtzite $\mathrm{ZnO}$ with a $d$-spacing of about $0.26 \mathrm{~nm}$. Furthermore, the major axes of these nanostructures were found to be parallel to the [0001] direction, indicating a predominant growth direction of [0001].

It is worth further noting in Figure 1d that the (100) peaks and (002) peaks for the twin-cone products obtained from the 


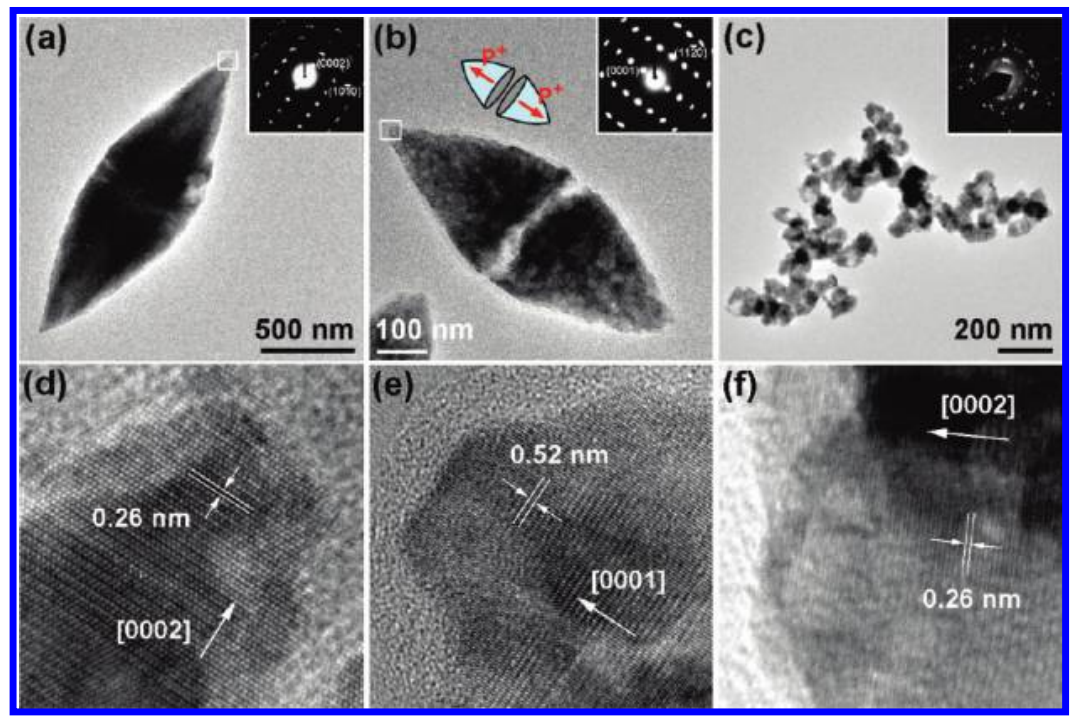

Figure 2. TEM images and SAED patterns of the $\mathrm{ZnO}$ nanostructure prepared with the antisolvent of (a) pure water, (b) $50 \%$ ethanol and $50 \%$ water, and (c) $90 \%$ ethanol and 10\% water. Their corresponding HRTEM images are shown in (d), (e), and (f), respectively. Injection time of $\mathrm{ZnO}$-containing DES $=5 \mathrm{~s}$.

pure water and $50 \%$ ethanol antisolvents were enhanced and suppressed, respectively, as compared with their relative intensities shown in the standard reference pattern. This phenomenon can be understood with the fact that these twin cones, deposited on a substrate for the XRD measurements, were preferentially lain sideways on the substrate with the axial direction, [001], parallel and the radial direction, [100], perpendicular to the substrate surfaces, as is evident from the SEM images of Figure 1a,b. During the XRD measurements, diffractions from the (100) and (001) planes were thus enhanced and suppressed, respectively, resulting in the relatively strong (100) and weak (002) peaks for curves (a) and (b) of Figure 1d, respectively. As for the $90 \%$ ethanol case, the XRD pattern, curve (c) of Figure 1d, complied with the standard reference pattern nicely because the granular product contained only a minor portion of twin-cone particles, and these twin-cone particles were randomly distributed, as shown in Figure 1c, giving no overall preferred orientation of the granular sample.

The formation of the twin-cone structure may be explained with the polar-field induced assembly concept proposed by Jia et al. for the formation of their double layer $\mathrm{ZnO}$ disks. ${ }^{22}$ Their double layer disks are similar in structural characteristics to our twin cones, with the double layer disks considered as a flattened version of our twin cones. Accordingly, a mechanism is proposed as follows. For each single cone, its round-bottom was formed first and then served as the base for the further growth along the major axis. ${ }^{23}$ For $\mathrm{ZnO},[0001]$ is the preferred growth direction because of the substantially larger surface energy of the (0001) plane. However, with the abundant product molecule supply achieved with the fast injection condition, the growth along the minor axes of the anisotropic product particles, the $\{10-10\}$ and $\{11-20\}$ planes, was not negligible. This would lead to an uneven, multi-dimensional growth of $\mathrm{ZnO}$ and, thus, the appearance of the cone-shape structure in the product.

The surface of the round bottom of each cone, which was perpendicular to the major axis of the cone, the [0001] direction, comprised the polar $\mathrm{O}^{2-}$-terminated $(000-1)$ planes of $\mathrm{ZnO}^{24}$ The apex surface of the cone, however, comprised the polar $\mathrm{Zn}^{2+}$-terminated planes. There, thus, was established a onedimensional polar field oriented from the $\mathrm{O}^{2-}$-terminated to $\mathrm{Zn}^{2+}$-terminated planes in each cone. ${ }^{25}$ According to the polarfield induced assembly concept proposed by Jia et al., to coun- terbalance the polar fields, two single cones with opposite polar field directions spontaneously assembled during the crystal growth process, ${ }^{22}$ leading to the formation of the twin-cone structures. This proposition also explained why fewer twin cones were found in the granular particle sample when $90 \%$ ethanol was used as the antisolvent. As evident from Figure $1 \mathrm{a}-\mathrm{c}$, when more ethanol was present in the antisolvent, the dimensions of the product particles were reduced. Because of the smaller dimension of the granular particles, the polar fields generated in each particle were weaker. Consequently, a less pronounced tendency of assembly between single particles was expected, leading to a fewer amount of twin cones in the granular particle sample. On the other hand, the much larger sizes of the product particles obtained from the pure water case resulted in much stronger polar fields and thus much stronger assembly between product particles. As a result, the slits between the two cones were not evident and TEM images were necessary to reveal their existence.

For the present antisolvent process, the morphology of the product $\mathrm{ZnO}$ can also be tuned by simply prolonging the injection time of the $\mathrm{ZnO}$-containing DES. Different from the results derived from the fast DES injection case, an injection time of $5 \mathrm{~s}$, the antisolvent process with a prolonged injection time of $5 \mathrm{~h}$, the slow injection condition, produced rod-shaped nanostructures. Figure 3 displays the morphology of the three $\mathrm{ZnO}$ products obtained with an injection time of $5 \mathrm{~h}$ using the three different antisolvents, pure water, 50\% ethanol, and $90 \%$ ethanol. As shown in Figure 3a, nanorods with a diameter of about $250 \mathrm{~nm}$ and lengths up to several $\mu \mathrm{m}$ were obtained when using pure water as the antisolvent. Increasing the ethanol content of the antisolvent to $50 \%$ did not cause apparent changes in the product morphology, only the dimensions, diameter, and length were slightly reduced. As for the $90 \%$ case, nanorod flowers were produced, as can be more clearly seen from the inset of Figure 3c. The constituent nanorods of the nanorod flowers were about $60 \mathrm{~nm}$ in diameter and 150-200 $\mathrm{nm}$ in length. The corresponding XRD analyses shown in Figure $3 \mathrm{~d}$ reveal again the wurtzite crystalline structure for the three nanorod-based products.

Typical TEM and HRTEM images taken on the three nanorod-based $\mathrm{ZnO}$ samples were shown in Figure 4. The dot patterns of the inset SAED images suggest the single crystallinity 


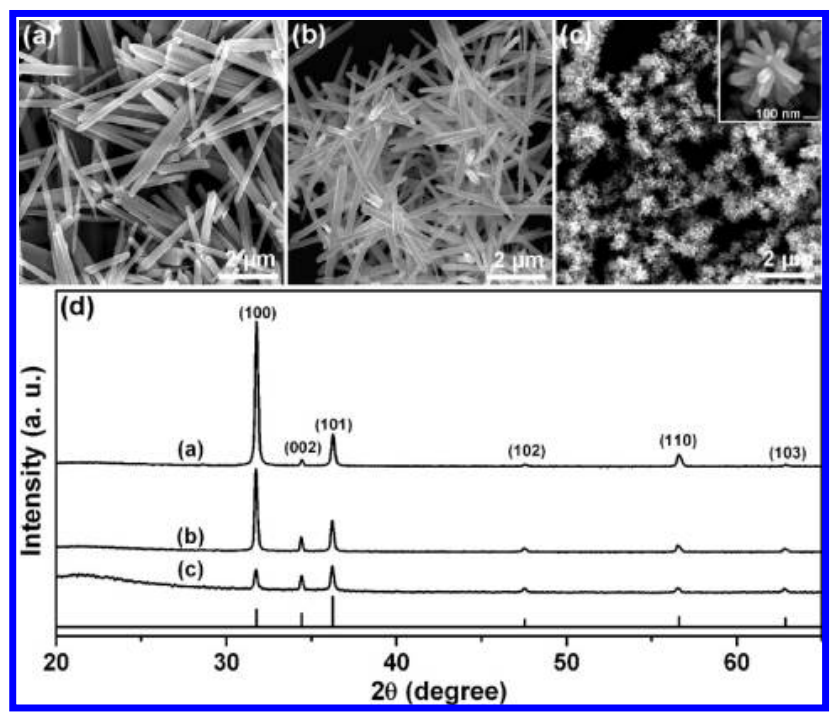

Figure 3. SEM images of $\mathrm{ZnO}$ nanostructures prepared with the antisolvent of (a) pure water, (b) 50\% ethanol and 50\% water, and (c) $90 \%$ ethanol and $10 \%$ water. The corresponding XRD patterns were shown in (d). Injection time of $\mathrm{ZnO}$-containing $\mathrm{DES}=5 \mathrm{~h}$.

of the nanorod products. Furthermore, the dots of the SAED images were not distorted (stretched) in shape, unlike those obtained for the twin-cone products prepared from the fast injection practice, indicating well crystallized nanorod samples. The corresponding HRTEM analyses revealed the (0002) lattice planes of wurtzite $\mathrm{ZnO}$ with a $d$-spacing of $0.26 \mathrm{~nm}$, as expected. In addition, the axes of the nanorods of all three $\mathrm{ZnO}$ samples were pointed in the [0001] direction, confirming that the asobtained $\mathrm{ZnO}$ nanorods were grown again along the [0001] direction. It is again worth noting that, in Figure 3d, the (100) peaks and (002) peaks for the nanorod products obtained from the pure water and 50\% ethanol antisolvents, were enhanced and suppressed, respectively, as compared with their relative intensities shown in the standard reference pattern. This phenomenon is the same with that observed and discussed for the twin-cone cases, only that it is more pronounced with the nanorod products because of the much stronger orientation effects associated with the nanorods. As for the $90 \%$ ethanol

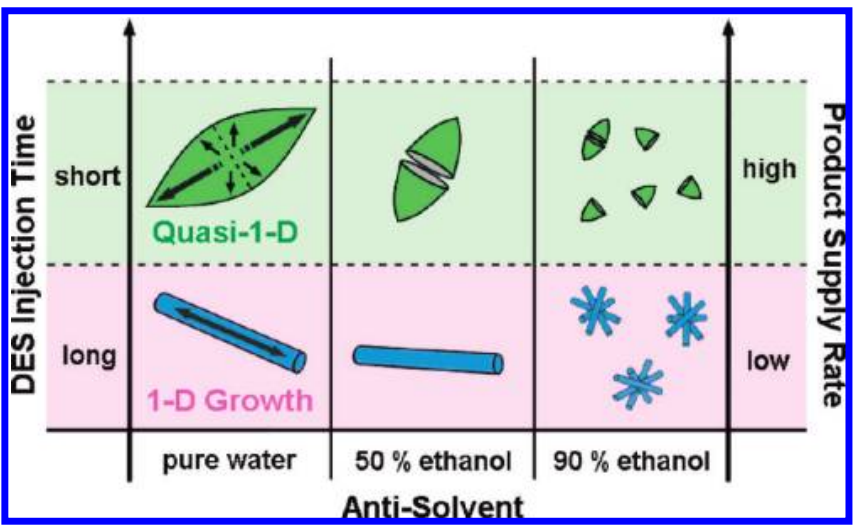

Figure 5. Schematic illustration of the effects of ethanol content and DES injection time on the morphology of the resulting $\mathrm{ZnO}$ nanostructures.

case, no overall preferred orientation of the nanorods was created because the nanorods were isotropically distributed in the nanorod flowers and the nanorod flowers were randomly packed on top of the substrate.

For the present antisolvent approach, the ethanol content of the antisolvent and the injection time of the $\mathrm{ZnO}$-containing DES are two key factors controlling the morphology of the resulting $\mathrm{ZnO}$ nanostructure. Figure 5 depicts a mechanism summary to clearly illustrate and explain these effects. With the introduction of ethanol into the antisolvent, a decrease in the dimension of the $\mathrm{ZnO}$ products was evident for both the fast and the slow injection cases. We suggested that the use of ethanol in the antisolvent may partly prohibit $\mathrm{ZnO}$ from being precipitated, which led to a lower growth rate of $\mathrm{ZnO}$ and, thus, the smaller product sizes. It should be noted that both urea and choline chloride of the DES possess higher solubility in water than in ethanol. The relevant solubility data were collected and listed in Table 1. As compared to ethanol, water is, thus, expected to have a stronger ability to catch DES molecules in the antisolvent process, giving a larger driving force for $\mathrm{ZnO}$ to be precipitated. Such driving forces, however, can be reduced by introducing ethanol into the antisolvent, which in turn suppressed the growth rate of $\mathrm{ZnO}$ and resulted in the production of $\mathrm{ZnO}$ with smaller dimensions. Moreover, the use of ethanol

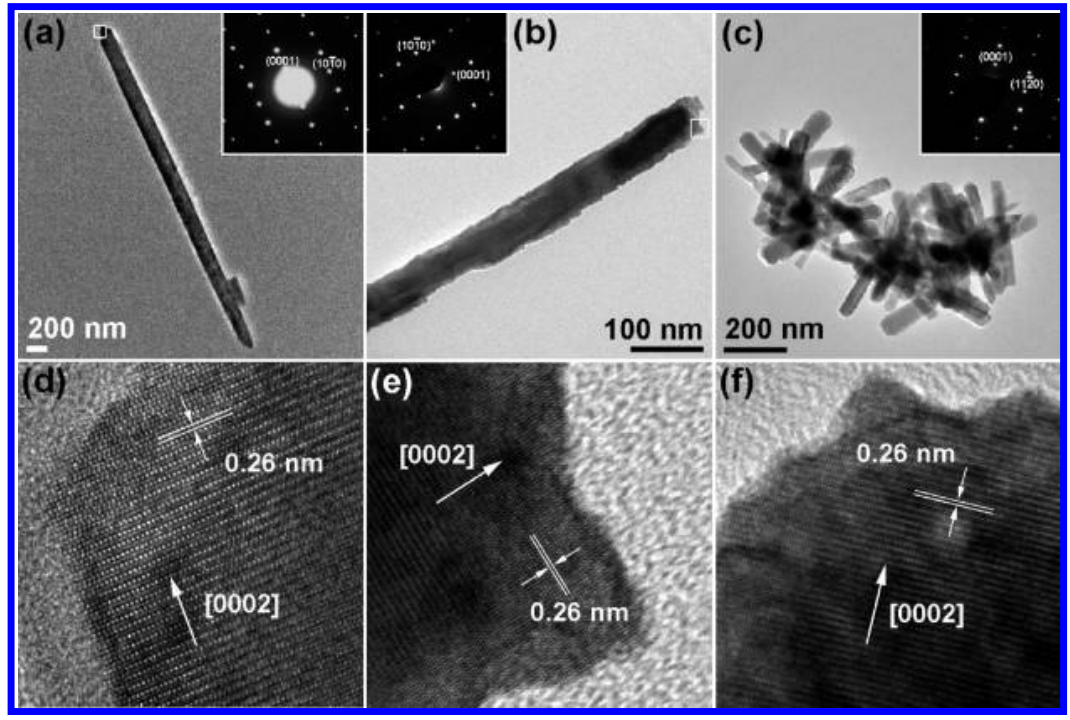

Figure 4. TEM images and SAED patterns of $\mathrm{ZnO}$ nanostructures prepared with the antisolvent of (a) pure water, (b) $50 \%$ ethanol and $50 \%$ water, and (c) $90 \%$ ethanol and $10 \%$ water. Their corresponding HRTEM images are shown in (d), (e), and (f), respectively. Injection time of ZnOcontaining DES $=5 \mathrm{~h}$. 
TABLE 1: Solubility of UCC Constituents in Water and in Ethanol

\begin{tabular}{lcc}
\hline & water & ethanol \\
\hline urea & $121 \mathrm{~g} / 100 \mathrm{~g}^{a}$ & $5.8 \mathrm{~g} / 100 \mathrm{~g}^{a}$ \\
choline chloride & $65 \mathrm{~g} / 100 \mathrm{~mL}^{b}$ & $22.5 \mathrm{~g} / 100 \mathrm{~mL}^{c}$
\end{tabular}

${ }^{a}$ Lee, F. M.; Lahti, L. E. J. Chem. Eng. Data 1972, 17, 304. ${ }^{b}$ OECD Safety Information Data. ${ }^{c}$ Measured in this work.

may alter the growth habit of $\mathrm{ZnO}$ because ethanol could readily adsorb onto the $\mathrm{Zn}^{2+}$-terminated (0001) planes. ${ }^{15}$ Because of this adsorption, a capping effect would emerge to inhibit $\mathrm{ZnO}$ from elongating along its $c$-axis in the crystal growth process. As a result, $\mathrm{ZnO}$ nanostructures with decreasing major-axis lengths were observed when increasing the ethanol content of the antisolvent. On the other hand, when prolonging the injection time from $5 \mathrm{~s}$ to $5 \mathrm{~h}$, a pronounced structural transformation of $\mathrm{ZnO}$ from twin cones to nanorods occurred. The significant difference in supply rate of the product $\mathrm{ZnO}$ molecules between the fast and the slow injection practices may account for this structural divergence. For crystal growth that leads to the formation of various nanostructures, the supersaturation degree developed in the system plays a determinant role. In general, to enable anisotropic growth to form nanowires or nanorods, the supersaturation degree of the growing species must be maintained below some critical value, above which twodimensional or even isotropic growth occurs. ${ }^{26}$ In other words, a limited supply of the growing material will result in predominant growth in the preferred directions, whereas an ample supply of the growing material is advantageous to competitive growth in less preferred directions. ${ }^{27}$ In the present antisolvent process, with a short injection time, a significant amount of $\mathrm{ZnO}$-containing DES was rapidly added into the antisolvent, thus, enabling competitive growth in the lesspreferred directions. In the slow injection process, the $\mathrm{ZnO}$ containing DES was slowly added into the antisolvent in small drops, limiting the supply of $\mathrm{ZnO}$ to favor one-dimensional growth in the most preferred direction.

The optical properties of the as-obtained $\mathrm{ZnO}$ products were further characterized with PL spectroscopy. Figure 6a shows the PL spectra of the three twin-cone based $\mathrm{ZnO}$ nanostructures from which three emission bands in the wavelength range of 350 to $600 \mathrm{~nm}$ were observed. The PL emission at about 385 $\mathrm{nm}$ (I) can be attributed to the typical excitonic band-to-band radiative emission of $\mathrm{ZnO}$ because of its near band edge location ( $368 \mathrm{~nm}$ corresponding to the energy bandgap of $3.37 \mathrm{eV}$ ). The minor shoulder emission band at $460 \mathrm{~nm}$ (II) was ever reported for $\mathrm{ZnO}$ nanorods, ${ }^{7}$ microrods,${ }^{28}$ nanotubes ${ }^{29}$ and flowerlike nanorods, ${ }^{30}$ but the mechanism for this blue emission is not yet clear. As to the green band at $540 \mathrm{~nm}$ (III), we ascribed it to the trap-state emission of $\mathrm{ZnO}$, which originated from the structural defects present in the products. ${ }^{30,31}$ This defect-level emission was even stronger than that of the excitonic emission for the pure water case, indicating the presence of a significant amount of defects in the products. This observation in fact complies with the phenomenon of stretched dots in the SEAD patterns for the defect existences discussed for Figure 2. Interestingly, a significant decrease in the intensity of the trapstate emission was found for samples prepared with increasing ethanol content in the antisolvents. This depression in the trapstate emission is a direct result of the decreasing defect density of the structure. Recall that the growth rate was decreased with increasing ethanol content in the antisolvent, leading to smaller dimensions of the products. During the recrystallization process, a low growth rate is advantageous to ensure formation of crystals

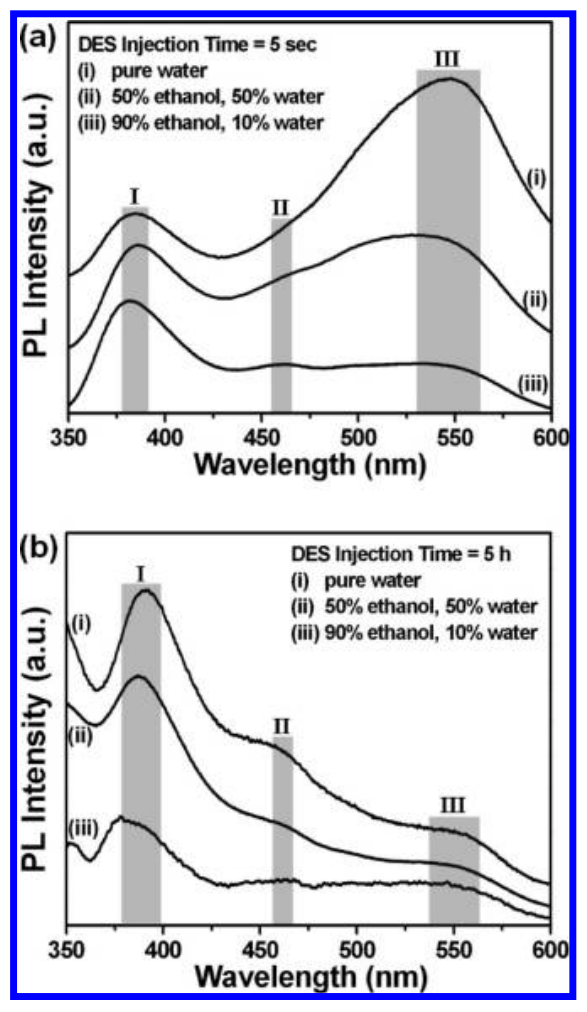

Figure 6. PL emission spectra for (a) twin-cone-based and (b) nanorodbased $\mathrm{ZnO}$ products.

with a low defect density, which then leads to a depression in the defect-related emission.

The PL spectra of the three nanorod-based $\mathrm{ZnO}$ samples were also studied and shown in Figure 6b. These samples exhibited similar PL characteristics with those of the twin-cone samples, having the three emission bands located at 385, 460, and 550 $\mathrm{nm}$. It is worth noting that, unlike the situation observed for the twin-cone cases, the excitonic emission at $385 \mathrm{~nm}$ was found to be dominant over the other two bands, indicating a better crystallinity, thus, less structural defects, for the three nanorod products. This observation is in good agreement with the better shaped dots of the SEAD patterns shown in Figure 4 for these nanorod products.

\section{Conclusions}

In conclusion, we have developed an environmentally friendly antisolvent approach for preparation of various $\mathrm{ZnO}$ nanostructures, including twin cones and nanorods, with controllable dimensions. The method was based on the dissolution of $\mathrm{ZnO}$ in the UCC DES, followed by the precipitation of $\mathrm{ZnO}$ nanostructures upon the extraction of the DES using an antisolvent. Through suitably modulating the two key processing conditions, the ethanol content of the antisolvent and the injection time of the $\mathrm{ZnO}$-containing DES, the morphology of the resulting $\mathrm{ZnO}$ nanostructures can be readily controlled. The presence of ethanol in the antisolvent lowered the product growth rate and thus decreased the product dimensions. If the injection rate of the $\mathrm{ZnO}$-containing DES was reduced, the supply of product $\mathrm{ZnO}$ molecules became limited and the crystal growth became more one-dimensional, dominated by the most preferred direction, turning product structure from twin cones to nanorods. The present study provides a new synthetic route to prepare anisotropic nanostructures of $\mathrm{ZnO}$ without the use of environmentally harmful chemicals, such as long-carbonchain surfactants and capping reagents, or severe reaction 
conditions, such as high temperature and high vacuum. The present method can be readily extended to produce versatile nanostructures of other functional materials.

Acknowledgment. This work was financially supported by the National Science Council, Taiwan, ROC (NSC-97-2221-E007-049 for D.S.-H.W., NSC-98-2113-M-009-015-MY2 for Y.J.H., and NSC-98-2221-E-007-034-MY3 for S.-Y.L.). J.-Y.D. extends special thanks to Mr. Cheng-Hsien Lin who provided many preliminary investigations and helpful suggestions.

\section{References and Notes}

(1) Haranath, D.; Sahai, S.; Joshi, P. Appl. Phvs. Lett. 2008, 92, 233113

(2) Huang, M. H.; Mao, S.; Feick, H.; Yan, H. Q.; Wu, Y. Y.; Kind, H.; Weber, E.; Russo, R.; Yang, P. D. Science 2001, 292, 1897.

(3) Kind, H.; Yan, H. Q.; Messer, B.; Law, M.; Yang, P. D. Adv. Mater. 2002, 14, 158 .

(4) Jang, E. S.; Won, J. H.; Hwang, S. J.; Choy, J. H. Adv. Mater. 2006, $18,3309$.

(5) Wang, Z. L.; Song, J. H. Science 2006, 312, 242.

(6) Wei, T. Y.; Yeh, P. H.; Lu, S. Y.; Wang, Z. L. J. Am. Chem. Soc. 2009, 131, 17690 .

(7) Wu, J. J.; Liu, S. C. Adv. Mater. 2002, 14, 215

(8) Kong, Y. C.; Yu, D. P.; Zhang, B.; Fang, W.; Feng, S. Q. Appl. Phys. Lett. 2001, 78, 407.

(9) Kong, X. Y.; Wang, Z. L. Nano Lett. 2003, 3, 1625.

(10) Kitano, M.; Hamabe, T.; Maeda, S.; Okabe, T. J. Cryst. Growth 1990, $102,965$.

(11) Vayssieres, L. Adv. Mater. 2003, 15, 464.

(12) Kahn, M. L.; Monge, M.; Colliere, V.; Senocq, F.; Maisonnat, A.; Chaudret, B. Adv. Funct. Mater. 2005, 15, 458.

(13) Yang, L. L.; Zhao, Q. X.; Willander, M.; Yang, J. H. J. Cryst. Growth 2009, 311, 1046.
(14) McLaren, A.; Valdes-Solis, T.; Li, G. Q.; Tsang, S. C. J. Am. Chem. Soc. 2009, 131, 12540.

(15) Pesika, N. S.; Hu, Z. S.; Stebe, K. J.; Searson, P. C. J. Phys. Chem. B 2002, 106, 6985 .

(16) Rogers, R. D.; Seddon, K. R. Ionic Liquids III: Fundamentals, Progress, Challenges, and Opportunities: American Chemical Society: Washington, DC, 2005.

(17) Ma, Z.; Yu, J. H.; Dai, S. Adv. Mater. 2010, 22, 261.

(18) Jastorff, B.; Stormann, R.; Ranke, J.; Molter, K.; Stock, F.; Oberheitmann, B.; Hoffmann, W.; Hoffmann, J.; Nuchter, M.; Ondruschka, B.; Filser, J. Green Chem. 2003, 5, 136.

(19) Abbott, A. P.; Capper, G.; Davies, D. L.; Rasheed, R. K.; Tambyrajah, V. Chem. Commun. 2003, 1, 70.

(20) Abbott, A. P.; Capper, G.; Davies, D. L.; Rasheed, R. K.; Shikotra, P. Inorg. Chem. 2005, 44, 6497.

(21) Rajan, K. Appl. Phvs. Lett. 1991, 58, 287.

(22) Jia, L. C.; Cai, W. P.; Wang, H. Q.; Zeng, H. B. Cryst. Growth Des. 2008, 8, 4367.

(23) Sun, X. F.; Qiu, X. Q.; Li, L. P.; Li, G. S. Inorg. Chem. 2008, 47, 4146.

(24) Wei, A.; Sun, X. W.; Xu, C. X.; Dong, Z. L.; Yang, Y.; Tan, S. T.; Huang, W. Nanotechnology 2006, 17, 1740.

(25) Wang, Z. L.; Kong, X. Y.; Zuo, J. M. Phvs. Rev. Lett. 2003, 91, 185502.

(26) Xia, Y. N.; Yang, P. D.; Sun, Y. G.; Wu, Y. Y.; Mayers, B.; Gates, B.; Yin, Y. D.; Kim, F.; Yan, Y. Q. Adv. Mater. 2003, 15, 353.

(27) Hsu, Y. J.; Lu, S. Y.; Lin, Y. F. Small 2006, 2, 268.

(28) Kale, R. B.; Lu, S. Y. J. Phvs.: Condens. Matter 2007, 19, 096209.

(29) Zhang, X. H.; Xie, S. Y.; Jiang, Z. Y.; Zhang, X.; Tian, Z. Q.; Xie, Z. X.; Huang, R. B.; Zheng, L. S. J. Phys. Chem. B 2003, 107, 10114. (30) Kale, R. B.; Hsu, Y. J.; Lin, Y. F.; Lu, S. Y. Solid State Commun. 2007, 142, 302.

(31) Sun, Y. M. Ph.D. Thesis, University of Science and Technology of China, Hefei, Anhue, People's Republic of China, 2000.

JP102396F 